\title{
ON LACUNARY DIRICHLET SERIES
}

\section{I. HIRSCHMAN, JR., AND J. A. JENKINS}

The following theorem is suggested by a result of S. Mandelbrojt $[2, \text { p. 101 }]^{1}$ concerning lacunary Fourier series.

ThEOREM 1. Let $f(s)=\sum_{k=1}^{\infty} a_{k} e^{-\lambda_{k^{e}}}$, where $0<\lambda_{1}<\lambda_{2}<\lambda_{3}<\cdots$, $\lim _{k \rightarrow \infty} \lambda_{k}=\infty$. We denote by $\gamma_{c}$ the abscissa of convergence of this series and by $\gamma_{a}$ the abscissa of analyticity. It is assumed that $\gamma_{c}<\infty, \gamma_{a}>-\infty$. Let $\nu<1$ be the exponent of convergence of the $\lambda_{k}$ 's and suppose that

$$
\left|f\left(s_{a}+\sigma\right)\right|=O\left[e^{-(1 / \sigma)^{\mu}}\right] \quad(\sigma \rightarrow 0+)
$$

where $s_{a}=\gamma_{a}+i \tau_{a}$. Then if $\mu>\nu /(1-\nu), f(s) \equiv 0$.

We shall in what follows prove a more general theorem including Theorem 1 as a special case. The methods of the present paper are closely related to and in part derived from the work of L. Schwartz [4]. However no appeal is made to other than standard theorems of analysis.

Let $m(0)=0$ and let $m(\sigma)$ be an increasing function defined for $0 \leqq \sigma \leqq a, a>0$. A function $f(s)$ which we may suppose analytic in the half-plane $\sigma>\gamma_{1}$ is said to have a zero of modular order $m(\sigma)$ at $s_{1}=\gamma_{1}+i \tau_{1}$ if

$$
\left|f\left(s_{1}+\sigma\right)\right| \leqq m(\sigma) \quad(0<\sigma \leqq b)
$$

for some $b>0$. Let us define $\sigma$ as a function of $\rho, \sigma=\eta(\rho)$, by the equation $e^{-\rho \sigma}=m(\sigma)$. Since $m(\sigma)$ decreases as $\sigma$ decreases to 0 this definition is effective. It is $\eta(\rho)$ which we shall use as the measure of the zero of $f(s)$. As an example, if $m(\sigma)=\exp \left[-\left(\sigma^{-\mu}\right)\right]$ then $\eta(\rho)=\rho^{-1 /(\mu+1)}$.

As the measure of the degree of lacunarity of our Dirichlet series we introduce

$$
\zeta(\rho)=\sum_{k=1}^{\infty} \log \left(1+\frac{2 \rho}{\lambda_{k}}\right) .
$$

If the sequence $\left\{\lambda_{k}\right\}_{1}^{\infty}$ has exponent of convergence $\nu$, then by a standard theorem on integral functions $[5$, p. 251],

$$
\zeta(\rho)=O\left(\rho^{\nu+\varepsilon}\right) \quad(\rho \rightarrow+\infty) .
$$

Presented to the Society, September 2, 1949; received by the editors April 11, 1949 and, in revised form, May 20, 1949.

${ }_{1}^{1}$ Numbers in brackets refer to the references cited at the end of the paper. 
We may now state our principal theorem.

Theorem 2. Let $0<\lambda_{1}<\lambda_{2}<\cdots, \sum_{1}^{\infty} \lambda_{z}^{-1}<\infty$, and let $\zeta(\rho)$ be defined as above. Let

$$
f(s)=\sum_{k=1}^{\infty} a_{k} e^{-\lambda_{k} s}
$$

have abscissa of convergence $\gamma_{c}<\infty$ and abscissa of analyticity $\gamma_{a}>-\infty$, and let $\gamma_{a}+i \tau_{a}$ be a zero of $f(s)$ of modular order $m(\sigma)$. Then if $\eta(\rho)$ is defined as above and if

$$
\liminf _{\rho \rightarrow \infty} \zeta(\rho)-\rho \eta(\rho)=-\infty,
$$

we have $f(s) \equiv 0$.

In the case considered in Theorem 1 we have, as we have seen, $\zeta(\rho)=O\left(\rho^{\nu+e}\right), \eta(\rho)=\rho^{-1 /(\mu+1)}$. The assumption $\mu>\nu /(1-\nu)$ shows that condition (1) is satisfied. Thus Theorem 2 does include Theorem 1.

It is clearly sufficient to prove our theorem for $\gamma_{a}=\tau_{a}=0$.

It is immediately verifiable that the function

$$
\frac{1}{(1+z)} \prod_{k=1, k \neq n}^{\infty}\left(\frac{\lambda_{k}+\rho-z}{\lambda_{k}+\rho+z}\right)=k_{n}(z, \rho) \quad(\rho>0)
$$

is analytic for $x \geqq 0$, and that

$$
\int_{-\infty}^{\infty}\left|k_{n}(x+i y, \rho)\right|^{2} d y \leqq \pi \quad(x \geqq 0) .
$$

By a simple extension of Plancherel's theorem, see $[3$, p. 8$]$, we see that if

$$
\phi(n, \rho, \sigma)=\underset{\substack{\operatorname{li.m} \\ \rightarrow \rightarrow \infty}}{\stackrel{(2)}{2 \pi i}} \int_{-i T}^{i T} \frac{k_{n}(z, \rho)}{k_{n}\left(\lambda_{n}+\rho, \rho\right)} e^{\sigma z} d z \quad(0 \leqq \sigma<\infty),
$$

then

$$
\int_{0}^{\infty} \phi(n, \rho, \sigma) e^{-\sigma z} d \sigma=\frac{k_{n}(z, \rho)}{k_{n}\left(\lambda_{n}+\rho, \rho\right)} \quad(x>0) .
$$

Further

$$
\|\phi(n, \rho, \sigma)\|_{2} \equiv\left[\int_{0}^{\infty}|\phi(n, \rho, \sigma)|^{2} d \sigma\right]^{1 / 2} \leqq\left[2^{1 / 2} k_{n}\left(\lambda_{n}+\rho, \rho\right)\right]^{-1}
$$

We assert that 

$\limsup _{\rho \rightarrow \infty}\|\phi(n, \rho, \sigma)\|_{2} e^{-\zeta(\rho)}<\infty$.

We have

$$
\begin{aligned}
\|\phi(n, \rho, \sigma)\|_{2} e^{-\zeta(\rho)} & {\left[\frac{1}{2^{1 / 2}} \prod_{k=1, k \neq n}^{\infty}\left(\frac{\lambda_{n}+\lambda_{k}+2 \rho}{\lambda_{k}-\lambda_{n}}\right)\right]\left[1+\lambda_{n}+\rho\right]\left[\prod_{k=1}^{\infty}\left(\frac{\lambda_{k}}{\lambda_{k}+2 \rho}\right)\right] } \\
= & \frac{1}{2^{1 / 2}}\left[\prod_{k=1, k \neq n}^{\infty}\left(1+\frac{\lambda_{n}}{\lambda_{k}+2 \rho}\right)\right]\left[\frac{1+\lambda_{n}+\rho}{\lambda_{n}+2 \rho} \lambda_{n}\right] \\
& \cdot\left[\prod_{k=1, k \neq n}^{\infty}\left(1-\frac{\lambda_{n}}{\lambda_{k}}\right)\right]^{-1} \quad(\rho \rightarrow \infty), \\
\sim & \frac{\lambda_{n}}{2^{3 / 2}}\left[\prod_{k=1, k \neq n}^{\infty}\left(1-\frac{\lambda_{n}}{\lambda_{k}}\right)\right]^{-1} \quad(\quad)
\end{aligned}
$$

which proves our assertion.

We define

$$
F(\rho, s)=e^{-\rho s} f(s)
$$

We assert that if

$$
\|F(\rho, \sigma)\|_{2} \equiv\left[\int_{0}^{\infty}|F(\rho, \sigma)|^{2} d \sigma\right]^{1 / 2},
$$

then

$$
\limsup _{\rho \rightarrow \infty}\|F(\rho, \sigma)\|_{2} e^{\rho \eta(\rho)}<\infty .
$$

Let $M$ be a bound for $f(\sigma)$ for $0<\sigma<\infty$. We have

$$
\begin{aligned}
\|F(\rho, \sigma)\|_{2} & \leqq\left[\int_{0}^{\eta(\rho)} e^{-2 \rho \sigma}|f(\sigma)|^{2} d \sigma\right]^{1 / 2}+\left[\int_{\eta(\rho)}^{\infty} e^{-2 \rho \sigma}|f(\sigma)|^{2} d \sigma\right]^{1 / 2} \\
& \leqq m(\eta(\rho))+M e^{-\rho \eta(\rho)} \\
& \leqq(M+1) e^{-\rho \eta(\rho)},
\end{aligned}
$$

which proves relation (4).

It follows from a well known theorem concerning restricted overconvergence of Dirichlet series, see $[1$, p. 141], that there exists an increasing sequence of integers $l_{k}$ for which

$$
\lim _{k \rightarrow \infty} \sum_{j=0}^{l_{k}} a_{j} e^{-\lambda_{j} \sigma}=f(\sigma)
$$


uniformly for $\epsilon \leqq \sigma<\infty$ for any $\epsilon>0$. Thus if $\epsilon>0$,

$$
\begin{aligned}
& \int_{0}^{\infty} \phi(n, \rho, \sigma) F(\sigma+\epsilon, \rho) d \sigma \\
&=\lim _{k \rightarrow \infty} \int_{0}^{\infty} \phi(n, \rho, \sigma) e^{-\rho(\sigma+\epsilon)} \sum_{j=1}^{l_{k}} a_{j} e^{-\lambda_{j}(\sigma+\epsilon)} d \sigma .
\end{aligned}
$$

By equation (2)

$$
\int_{0}^{\infty} \phi(n, \rho, \sigma) e^{-\rho(\sigma+\epsilon)} a_{j} e^{-\lambda_{j}(\sigma+\epsilon)} d \sigma= \begin{cases}0, & j \neq n, \\ e^{-\epsilon \rho-\epsilon \lambda_{n}} a_{n}, & j=n .\end{cases}
$$

Hence

$$
\int_{0}^{\infty} \phi(n, \rho, \sigma) F(\rho, \sigma+\epsilon) d \sigma=e^{-\epsilon \rho-\epsilon \lambda_{n}} a_{n}
$$

By Schwarz's inequality

$$
\left|a_{n}\right| \leqq e^{\epsilon \rho+e \lambda_{n}}\|\phi(n, \rho, \sigma)\|_{2}\|F(\sigma+\epsilon, \rho)\|_{2} \text {. }
$$

If $\epsilon$ is allowed to approach zero through positive values, we obtain

$$
\left|a_{n}\right| \leqq\|\phi(n, \rho, \sigma)\|_{2}\|F(\sigma, \rho)\|_{2} \text {. }
$$

Using equations (3) and (4) we see that

$$
\log \left|a_{n}\right| \leqq \zeta(\rho)-\rho \eta(\rho)+C
$$

where $C$ is a constant which depends on $\boldsymbol{n}$ but not upon $\rho$. If we allow $\rho$ to increase without limit, assumption (1) of Theorem 2 implies that

$$
\log \left|a_{n}\right|=-\infty, \quad \text { that is, } a_{n}=0 \text {. }
$$

Since this holds for each $n=1,2, \cdots, f(s) \equiv 0$ and our theorem is proved.

We shall now prove that if assumption (1) is replaced by the weaker assumption

$$
\lim _{\rho \rightarrow \infty} \inf \operatorname{c\zeta }(\rho)-\rho \eta(\rho)=-\infty
$$

where $c<2^{-3 / 2}$, then Theorem 2 is false.

Let us take for the constants $\lambda_{k}, 0<a<b<1<4<9<\ldots$. As in Titchmarsh $[5$, p. 271] we find that

$$
\log \left[\prod_{1}^{\infty}\left(1+\frac{x}{k^{2}}\right)\right] \sim \pi x^{1 / 2} \quad(x \rightarrow \infty) .
$$


It follows that

$$
\begin{aligned}
\zeta(\rho)= & \log \left(1+\frac{2 \rho}{a}\right)+\log \left(1+\frac{2 \rho}{b}\right) \\
& +\sum_{k=1}^{\infty} \log \left(1+\frac{2 \rho}{k^{2}}\right) \sim \pi(2 \rho)^{1 / 2} \quad(\rho \rightarrow \infty) .
\end{aligned}
$$

We define

$$
f(s)=\frac{1}{2 \pi i} \int_{-i \infty}^{i \infty} \frac{e^{s z}}{\left(1+\frac{z}{a}\right)\left(1+\frac{z}{b}\right) \prod_{k=1}^{\infty}\left(1+\frac{z}{k^{2}}\right)} d z .
$$

Deforming the line of integration of this integral to $\mathrm{Rl} z=-(n+1 / 2)^{2}$ and allowing $n$ to approach infinity we obtain for $s$ real and positive an expansion of $f(s)$ into a Dirichlet series with exponents $a, b, 1$, $4,9, \cdots$ which then converges in the half-plane Rl $s>0$. The details of this are left to the reader. Again deforming the line of integration, this time to $\mathrm{Rl} z=(\pi / 2 \sigma)^{2}$, and using equation (5), we see that if $\epsilon>0$ is fixed, then for $\sigma>0$ sufficiently small we have

$$
|f(\sigma)| \leqq \exp \left[-\frac{1-2 \epsilon}{4} \frac{\pi^{2}}{\sigma}\right] M(\sigma)
$$

where

$$
\begin{aligned}
M(\sigma) & =\frac{1}{2 \pi} \int_{\mathrm{R} 1 z-(x / 2 \sigma)^{2}}\left|\frac{d z}{\left(1+\frac{z}{a}\right)\left(1+\frac{z}{b}\right)}\right| \\
& =o(1)
\end{aligned}
$$

Thus we may associate with $f(s)$ and the origin the modular order $m(\sigma)=\exp \left[-((1-2 \epsilon) / 4)\left(\pi^{2} / \sigma\right)\right]$. We immediately find that

$$
\eta(\rho)=\frac{\pi}{2}(1-2 \epsilon)^{1 / 2} \rho^{-1 / 2}
$$

Comparing equations (6) and (8), we deduce that Theorem 2 is false if $c<2^{-3 / 2}$, which is what we wished to show.

\section{REFERENCES}

1. V. Bernstein, Leçons sur les progrès rêcents de la théorie des series de Dirichlet, Paris, 1933.

2. S. Mandelbrojt, Analytic functions and classes of infinitely differentiable func- 
tions, The Rice Institute Pamphlet, vol. 29, 1942.

3. R. Paley and N. Wiener, Fourier transforms in the complex domain, New York, 1934.

4. L. Schwartz, Etudes des sommes d'exponentielles reelles, Paris, 1943.

5. E. Titchmarsh, The theory of functions, 2d ed., Oxford, 1939.

HARVARD UNIVERSITY

\section{ON THE ABSOLUTE CONVERGENCE OF TRIGONOMETRICAL SERIES}

TAMOTSU TSUCHIKURA AND SHIGEKI YANO

1. Theorem 1. Suppose that a trigonometrical series

$$
\sum \rho_{n} \cos \left(n x-\alpha_{n}\right) \quad\left(\rho_{n} \geqq 0, n=1,2, \cdots\right)
$$

and its conjugate series

$$
\sum \rho_{n} \sin \left(n x-\alpha_{n}\right)
$$

are convergent absolutely at $x=x_{0}$ and $x=x_{1}$, respectively. If

$$
x_{1}-x_{0}=p \pi / q \quad(p / q \text { irreducible })
$$

where $p$ is an integer positive, negative, or zero, and $q$ is an odd integer, then

$$
\sum \rho_{n}<\infty \text {. }
$$

We shall see in Theorem 2 that the above theorem is no longer true if $p / q$ is replaced by $p^{\prime} / q^{\prime}$ with an even $q^{\prime}$ and $p^{\prime} \neq 0$, or by an irrational number.

Proof of Theorem 1. If we put $x_{1}-x_{0}=p \pi / q \equiv h$, then by Fatou's theorem $^{1}$

$$
\sum \rho_{n}\left|\cos \left(n\left(x_{1}-s h\right)-\alpha_{n}\right)\right|<\infty
$$

for every odd $s$. Hence from the identity

$$
\begin{aligned}
\sin \left(n x_{1}-\alpha_{n}\right)= & \cos n s h \sin \left(n\left(x_{1}-s h\right)-\alpha_{n}\right) \\
& +\sin n s h \cos \left(n\left(x_{1}-s h\right)-\alpha_{n}\right)
\end{aligned}
$$

we deduce immediately that

$$
\left.\sum \rho_{n} \mid \cos n s h \sin \left(n\left(x_{1}-s h\right)\right)-\alpha_{n}\right) \mid<\infty .
$$

Received by the editors October 18, 1948 and, in revised form, May 14, 1949.

1 See, for example, A. Zygmund, Trigonometrical series, p. 134. 